\title{
A NOTE ON CONTINUOUS MAPPINGS AND THE PROPERTY OF J. L. KELLEY
}

\author{
HISAO KATO
}

(Communicated by James E. West)

\begin{abstract}
In this paper, it is proved that if $X$ is a continuum and $\omega$ is any Whitney map for $C(X)$, then the following are equivalent:

(1) $X$ has property $[\mathrm{K}]$

(2) There exists a (continuous) mapping $F: X \times I \times[0, \omega(X)] \rightarrow C(X)$ such that $F(\{x\} \times I \times\{t\})=\left\{A \in \omega^{-1}(t) \mid x \in A\right\}$ for each $x \in X$ and $t \in[0, \omega(X)]$, where $I=[0,1]$.

(3) For each $t \in[0, \omega(X)]$, there is an onto map $f: X \times I \rightarrow \omega^{-1}(t)$ such that $f(\{x\} \times I)=\left\{A \in \omega^{-1}(t) \mid x \in A\right\}$ for each $x \in X$. Some corollaries are obtained also.
\end{abstract}

\section{INTRODUCTION}

By a continuum we mean a compact connected metric space. For a continuum $X, C(X)$ denotes the hyperspace of all nonempty subcontinua of $X$, with the topology induced by the Hausdorff metric $d_{H}$. Then the hyperspace $C(X)$ is a continuum, and in fact, is pathwise connected. In [6, 2.7. Theorem], Kelley proved that $C(X)$ is the continuous image of the Cantor fan, i.e., the cone over the Cantor set.

In this paper, we prove the following: Let $X$ be a continuum and $\omega$ be any Whitney map for $C(X)$. Then the following are equivalent:

(1) $X$ has property $[\mathrm{K}]$.

(2) There exists a map $F: X \times I \times[0, \omega(X)] \rightarrow C(X)$ such that

$$
F(\{x\} \times I \times\{t\})=\omega_{x}^{-1}(t)
$$

for each $x \in X$, where $\omega_{x}^{-1}(t)=\left\{A \in \omega^{-1}(t) \mid x \in A\right\}$.

(3) For each $t \in[0, \omega(X)]$, there is an onto map $f: X \times I \rightarrow \omega^{-1}(t)$ such that $f(\{x\} \times I)=\omega_{x}^{-1}(t)$ for each $x \in X$.

Received by the editors April 18, 1988.

1980 Mathematics Subject Classification (1985 Revision). Primary 54B20, 54C65; Secondary $54 \mathrm{C} 05,54 \mathrm{C} 60$.

Key words and phrases. Hyperspaces of continua, Whitney map, property $[\mathrm{K}]$, continuous selection, weakly chainable, uniformly pathwise connected. 
Note that if $F$ is a map that satisfies (2) as above, then $F(\{x\} \times I \times\{0\})=\{x\}$ for each $x \in X$ and $F \mid X \times I \times\{t\} \rightarrow \omega^{-1}(t)$ is surjective. It is known that the hyperspace $C(X)$ is contractible if and only if there is a map $G: X \times$ $[0, \omega(X)] \rightarrow C(X)$ such that $G(x, t) \in \omega_{x}^{-1}(t)$ for each $x \in X$ and $t \in$ $[0, \omega(X)]$, where $\omega$ is any Whitney map for $C(X)$ (see [6]).

To prove the above theorem, we use a selection theorem of D. W. Curtis [2, Theorem 2.2] and an idea of S. Ferry [4, the proof of 3.1]. As a corollary, the property of being weakly chainable (or uniformly pathwise connected) is a Whitney property for the class of continua that have property $[\mathrm{K}]$. The first is a partial answer to Roger's problem [11, pp. 384, 112]. Also, the following problem is considered: Is it true that the property $[\mathrm{K}]$ is a Whitney property?

We refer the reader to Nadler's monograph [13] for hyperspace theory.

\section{Preliminaries}

Let $X$ be a continuum. A map $\omega: C(X) \rightarrow[0, \infty)$ is said to be a Whitney map for $C(X)$ provided that $\omega$ satisfies the following conditions:

(1) $\omega(\{x\})=0$ for each $x \in X$ and

(2) if $A, B \in C(X), A \subset B$, and $A \neq B$, then $\omega(A)<\omega(B)$.

In [15] Whitney proved that there always exists a Whitney map on any continuum. Then $\omega^{-1}(t) \quad(0 \leq t<\omega(X))$ is a continuum and it is called a Whitney continuum. Let $X$ be a continuum with metric $d$. Then $X$ has property $[\mathrm{K}]$ [6] if for every $\varepsilon>0$ there exists $\delta>0$ such that, for every pair of points $x, y$ of $X$ with $d(x, y)<\delta$ and every subcontinuum $A$ containing $x$, there exists a subcontinuum $B$ containing $y$ with $d_{H}(A, B)<\varepsilon$. In [14] Wardle proved that a continuum $X$ has property $[\mathrm{K}]$ if and only if the set-valued function $\alpha: X \rightarrow C(X)$, where $\alpha(x)=\{A \in C(X) \mid x \in A\}$, is continuous. We may assume that $X$ is naturally contained in $C(X)$.

Let $(Y, d)$ be a metric space, and for each positive integer $n$ let $P_{n}=\left\{\left(t_{i}\right) \in\right.$ $\left.I^{n} \mid \sum_{i=1}^{n} t_{i}=1\right\}$. A convex structure on $(Y, d)[2,2.1]$ is a sequence of subsets $M_{n} \subset Y^{n}$ and maps $k_{n}: M_{n} \times P_{n} \rightarrow Y$ satisfying the following conditions:

$$
\begin{gathered}
k_{n}\left(y, \ldots, y ; t_{1}, \ldots, t_{n}\right)=y ; \\
k_{n}\left(y_{1}, y_{2}, \ldots, y_{n} ; t_{1}, t_{2}, \ldots, t_{i-1}, 0, t_{i+1}, \ldots, t_{n}\right) \\
=k_{n-1}\left(y_{1}, \ldots, y_{i-1}, y_{i+1}, \ldots, y_{n} ; t_{1}, \ldots, t_{i-1}, t_{i+1}, \ldots, t_{n}\right) ;
\end{gathered}
$$

(C3) for each $\varepsilon>0$ there is $\delta>0$ such that for every $n$ and $\left(t_{i}\right) \in P_{n}$,

$$
d\left(k_{n}\left(\left(y_{i}\right) ;\left(t_{i}\right)\right), k_{n}\left(\left(y_{i}^{\prime}\right) ;\left(t_{i}\right)\right)<\varepsilon \text { if } d\left(y_{i}, y_{i}^{\prime}\right)<\delta \text { for each } i\right. \text {. }
$$

A subset $C$ of $Y$ is convex if for each $n, C^{n} \subset M_{n}$ and $k_{n}\left(C^{n} \times P_{n}\right) \subset C$. In $[2,2.2$. Theorem] Curtis proved the following selection theorem:

(1.1) Theorem (D. W. Curtis). Let $X$ be paracompact, $(Y, d)$ a metric space with a convex structure, and $\Phi: X \rightarrow Y$ a lower semicontinuous set-valued 
function, with each $\Phi(x)$ a complete, convex subset of $Y$. Then $\Phi$ admits a continuous selection $s: X \rightarrow Y$.

\section{RESUlt}

In this section, we prove the following main result of this paper:

(2.1) Theorem. Let $X$ be a continuum and $\omega$ be a Whitney map for $C(X)$. Then the following are equivalent:

(1) $X$ has property $[\mathrm{K}]$.

(2) There exists $F: X \times I \times[0, \omega(X)] \rightarrow C(X)$ such that $F(\{X\} \times I \times\{t\})$ $=\omega_{x}^{-1}(t)$ for each $x \in X$ and $t \in[0, \omega(X)]$, where $\omega_{x}^{-1}(t)=\left\{A \in \omega^{-1}(t) \mid x \in\right.$ $A\}$.

(3) For each $t \in[0, \omega(X)]$, there is an onto map $f: X \times I \rightarrow \omega^{-1}(t)$ such that $f(\{x\} \times I)=\omega_{x}^{-1}(t)$ for each $x \in X$.

To prove (2.1) we need the following:

(2.2) Theorem cf. [7, (2.3)]). Let $X$ be a continuum and $\omega$ be any Whitney map for $C(X)$. Then for any $\varepsilon>0$ there is $\delta>0$ such that if $A, B \in C(X)$, $|\omega(A)-\omega(B)|<\delta$ and $B \subset U(A, \delta)$, then $d_{H}(A, B)<\varepsilon$, where $U(A, \delta)$ denotes the $\delta$-neighborhood of $A$ in $X$.

A map $f: X \rightarrow B$ between metric spaces is said to be strongly regular if $f$ is proper and if for each $b \in B$ and $\varepsilon>0$ there is $\delta>0$ such that if $d\left(b, b^{\prime}\right)<$ $\delta$, then there are maps $g_{b b^{\prime}}: f^{-1}(b) \rightarrow f^{-1}\left(b^{\prime}\right)$ and $g_{b^{\prime} b}: f^{-1}\left(b^{\prime}\right) \rightarrow f^{-1}(b)$ and homotopies $h_{t}: f^{-1}(b) \rightarrow f^{-1}(b)$ and $k_{t}: f^{-1}\left(b^{\prime}\right) \rightarrow f^{-1}\left(b^{\prime}\right)$ such that

(i) $d\left(g_{b b^{\prime}}(x), x\right)<\varepsilon$ and $d\left(h_{t}(x), x\right)<\varepsilon$ for all $x \in f^{-1}(b)$ and $0 \leq t \leq$ 1

(ii) $d\left(g_{b^{\prime} b}(x), x\right)<\varepsilon$ and $d\left(k_{t}(x), x\right)<\varepsilon$ for all $x \in f^{-1}\left(b^{\prime}\right)$ and $0 \leq$ $t \leq 1$

(iii) $h_{0}=g_{b^{\prime} b} \cdot g_{b b^{\prime}}$ and $h_{1}=\mathrm{id}$,

(iv) $k_{0}=g_{b b^{\prime}} \cdot g_{b^{\prime} b}$ and $k_{1}=$ id.

(2.3) Lemma. Let $\Lambda(X) \subset C(C(X))$ denote the space of maximal order arcs in $C(X)$, and let $e: \Lambda(X) \rightarrow X$ be the map defined by $e(\alpha)=\alpha(0)$ for $\alpha \in \Lambda(X)$ (see [2]). If $X$ has property $[\mathrm{K}]$, then $e$ is a strongly regular mapping.

Proof. Let $\omega$ be a Whitney map for $C(X)$. Also, let $\varepsilon>0$ and $x_{0} \in X$. By (2.2) there exist open subsets $U_{1}^{*}, U_{2}^{*}, \ldots, U_{n}^{*}$ of $\Lambda(X)$ such that $\cup U_{i}^{*} \supset$ $e^{-1}\left(x_{0}\right)$ and diam $U_{i}^{*}<\varepsilon / 2$ for each $i$, where $U_{i}^{*}$ is of the following form:

$$
\begin{gathered}
U_{i}^{*}=\left\{\alpha \in \Lambda(X) \mid \alpha\left(t_{j}^{i}\right) \subset V_{j}^{i} \text { for some } 0 \leq t_{0}^{i}<t_{1}^{i}<\cdots<t_{n_{i}}^{i}\right. \\
\left.\leq \omega(X) \text { and open subsets } V_{0}^{i}, V_{1}^{i}, \ldots, V_{n_{i}}^{i} \text { of } X\right\}, \\
\text { where } \alpha\left(t_{j}^{i}\right) \in \alpha \text { with } \omega\left(\alpha\left(t_{j}^{i}\right)\right)=t_{j}^{i} .
\end{gathered}
$$


Let $W_{i}^{*}=\left\{\alpha \in \Lambda(X) \mid \alpha\left(t_{j}^{i}\right) \subset \mathrm{Cl} V_{j}^{i}\right.$ for each $\left.j=0,1, \ldots, n_{i}\right\}$. Note that $W_{i}^{*}$ is closed in $\Lambda(X)$ and $U_{i}^{*} \subset W_{i}^{*}$. Note that if $V^{i}(i=1,2, \ldots$,$) is a$ neighborhood of $x_{0}$ such that $\mathrm{Cl} V^{i+1} \subset V^{i}, \cap \mathrm{Cl} V^{i}=\left\{x_{0}\right\}$, and $U_{i}^{*}=\{\alpha \in$ $\left.\Lambda(X) \mid \alpha(0) \in V^{i}\right\}$, then $\cap W_{i}^{*}=e^{-1}\left(x_{0}\right)$.

We may assume that diam $W_{i}^{*}<\varepsilon$ and $\bigcap_{j \in J} U_{j}^{*}\left(x_{0}\right)=\varnothing$ if and only if $\bigcap_{j \in J} U_{j}^{*} \neq \varnothing$ if and only if $\bigcap_{j \in J} W_{j}^{*} \neq \varnothing$, where $J$ is a subset of $\{0,1, \ldots, n\}$ and $U_{i}^{*}\left(x_{0}\right)=e^{-1}\left(x_{0}\right) \cap U_{i}^{*}$. Since $e$ is an open map, there is a neighborhood $O$ of $x_{0}$ in $X$ such that if $x \in O$, then $e^{-1}(x) \subset \cup U_{i}^{*}$ and $e^{-1}(x) \cap \bigcap_{j \in J} U_{j}^{*} \neq \varnothing$ if and only if $\bigcap_{j \in J} U_{j}^{*}\left(x_{0}\right) \neq \varnothing$. For each $x \in O$, let $W_{i}^{*}(x)=e^{-1}(x) \cap W_{i}^{*}$.

We show that $W_{i}^{*}(x)$ is convex. Consider $\alpha_{1}, \alpha_{2}, \ldots, \alpha_{n} \in e^{-1}(x)$ and $\left(t_{1}, t_{2}, \ldots, t_{n}\right) \in P_{n}$. Suppose first that each $t_{i}>0$. Define $k_{n}\left(\alpha_{1}, \alpha_{2}, \ldots, \alpha_{n}\right.$; $\left.t_{1}, t_{2}, \ldots, t_{n}\right)=\alpha$, where $\alpha=\left\{\alpha_{1}\left(\tau_{1}\right) \cup \cdots \cup \alpha_{i-1}\left(\tau_{i-1}\right) \cup \alpha_{i}(t) \mid 1 \leq i \leq n\right.$, $\left.0 \leq t \leq \tau_{i}\right\} \subset C(X)$ and $\tau_{i}=t_{i} /\left(t_{i}+\cdots+t_{n}\right)$. In the case that $t_{i}=0$ for some $i, k_{n}\left(\alpha_{1}, \alpha_{2}, \ldots, \alpha_{n} ; t_{1}, t_{2}, \ldots, t_{n}\right)$ is defined by the boundary condition (C2) (see the proof of [2, (4.1)]). Clearly, if $\alpha_{1}, \alpha_{2}, \ldots, \alpha_{n} \in W_{i}^{*}(x)$, then $k_{n}\left(\alpha_{1}, \alpha_{2}, \ldots, \alpha_{n} ; t_{1}, t_{2}, \ldots, t_{n}\right) \in W_{i}^{*}(x)$ and $\left\{k_{n}\right\}$ satisfies all the conditions for a convex structure. Hence $W_{i}^{*}(x)$ is convex, in particular, an AR. If $\bigcap_{j \in J} W_{j}^{*}(x) \neq \varnothing$ for a subset $J$ of $\{0,1, \ldots, n\}$, then $\bigcap_{j \in J} W_{j}^{*}(x)$ is an AR. Note that $\bigcap_{j \in J} W_{j}^{*}\left(x_{0}\right) \neq \varnothing$ if and only if $\bigcap_{j \in J} W_{j}^{*}(x) \neq \varnothing$. Hence we can see that there is a map $f: e^{-1}(x) \rightarrow e^{-1}\left(x_{0}\right)$ such that $f\left(W_{i}^{*}(x)\right) \subset W_{i}^{*}\left(x_{0}\right)$ for each $i$. Also, there is a map $g: e^{-1}\left(x_{0}\right) \rightarrow e^{-1}(x)$ such that $g\left(W_{i}^{*}\left(x_{0}\right)\right) \subset$ $W_{i}^{*}(x)$ for each $i$. Clearly, $f \circ g\left(W_{i}^{*}\left(x_{0}\right)\right) \subset W_{i}^{*}\left(x_{0}\right)$ and $g \cdot f\left(W_{i}^{*}(x)\right) \subset W_{i}^{*}(x)$ for each $i$. We can easily see that $f$ and $g$ satisfy the desired conditions. Thus $e: \Lambda(X) \rightarrow X$ is a strongly regular mapping with AR fibers.

Proof of (2.1). First, we prove that (1) implies (2). We use Ferry's idea as in the proof of [4, 3.1]. In [2] it was proved that $\Lambda(X)$ has a convex structure. By (2.3) $e$ is a strongly regular mapping with AR fibers. Note that $e^{-1}(x)$ is convex (see [2]). Since $\Lambda(X)$ is a compact metric space, it can be embedded in the Hilbert cube $Q=[0,1]^{\infty}$. Let $F(Q, \Lambda(X))$ be the space of maps from $Q$ to $\Lambda(X)$ in the sup norm and let $H \subset F(Q, \Lambda(X))$ be the subspace $\{g \mid g$ retracts $Q$ onto some $\left.e^{-1}(x)\right\}$. Note that $H$ is complete.

Define a convex structure on $H$ as follows: Suppose that $M_{n} \subset \Lambda(X)^{n}$ and $k_{n}: M_{n} \times P_{n} \rightarrow \Lambda(X)$ satisfy the conditions of convex structure on $\Lambda(X)$. By $[2,4.1] e^{-1}(x)$ is a convex subset for each $x \in X$. Let $q: H \rightarrow X$ be the map defined by $q(r)=x$, if $r$ retracts $Q$ onto $e^{-1}(x)$. Let $M_{n}^{*}=\left\{\left(r_{1}, \ldots, r_{n}\right) \in\right.$ $\left.H^{n} \mid q\left(r_{1}\right)=\cdots=q\left(r_{n}\right)\right\}$ and let $k_{n}^{*}: M_{n}^{*} \times P_{n} \rightarrow H$ be the map defined by $k_{n}^{*}\left(r_{1}, \ldots, r_{n} ; t_{1}, \ldots, t_{n}\right)(z)=k_{n}\left(r_{1}(z), \ldots, r_{n}(z) ; t_{1}, \ldots, t_{n}\right)$ for each $z \in$ $Q$. Clearly, $H$ has a convex structure. By [4, Step I, p. 376] $q$ is an open map. Since $q^{-1}(x)$ is convex in $H$, by (1.1) there is a section $s: X \rightarrow H$ of $q$, i.e., $q \circ s=\mathrm{id}_{X}$. 
Define a map $G: X \times Q \rightarrow \Lambda(X)$ by $G(x, z)=s(x)(z)$ for each $x \in X$ and $z \in Q$. Define a map $G^{\prime}: X \times Q \times[0, \omega(X)] \rightarrow C(X)$ by $G^{\prime}(x, z, t)=$ $G(x, z) \cap \omega^{-1}(t)$. Finally, choose an onto map $h: I \rightarrow Q$ and then define $F: X \times I \times[0, \omega(X)] \rightarrow C(X)$ by $F(x, s, t)=G^{\prime}(x, h(s), t)$. Clearly, $F$ satisfies the desired conditions.

Clearly, (2) implies (3). Also, (3) implies (1) (see [13, (16.14)]).

\section{SOME COROLLARIES}

In this section, we give some applications of $(2.1)$. In [10,3] Lelek and Fearnley respectively defined the notion "weakly chainable," and they proved that a continuum $X$ is weakly chainable if and only if $X$ is a continuous image of the pseudo-arc. Clearly, the product of two weakly chainable continua is weakly chainable. By $(2.1)$ we have:

(3.1) Corollary. The property of being weakly chainable is a Whitney property for the class of continua that have property $[\mathrm{K}]$.

(3.1) is a partial answer to Rogers problem [11, p. 384].

In [9] Kuperberg defined the notion "uniformly pathwise connected" and he proved that a continuum $X$ is uniformly pathwise connected if and only if $X$ is a continuous image of the Cantor fan. By $[9,(3.7)]$ we easily see that the product of two uniformly pathwise connected continua is uniformly pathwise connected. Hence we have:

(3.2) Corollary. The property of being uniformly pathwise connected is a Whitney property for the class of continua that have property $[\mathrm{K}]$.

In $[12$, p. $558 ; 14$, p. 295], Nadler and Wardle respectively asked the following problem:

If a continuum $X$ has property $[\mathrm{K}]$, is it true that $C(X)$ or $\omega^{-1}(t)$ has property $[\mathrm{K}]$ ?

(See [5] for a partial answer.) Here, we consider the following problem:

If a continuum $X$ has property $[\mathrm{K}]$, is it true that $X \times I$ has property $[\mathrm{K}]$ ?

Then we have:

(3.3) Corollary. If problem (**) has an affirmative answer, then problem (*) has an affirmative answer.

Proof. Suppose that (**) has an affirmative answer. Then $X \times I^{n}$ has property [K]. Now we show that $X \times Q$ has property [K]. Let $\varepsilon>0$. Consider the projection $p_{n}: X \times Q \rightarrow X \times I^{n}$. For some integer $n, p_{n}$ is a monotone and $\varepsilon / 2$ mapping. Choose $\delta>0$ such that if $A$ is a subset of $X \times I^{n}$ with diam $A<\delta$, then $\operatorname{diam} p_{n}^{-1}(A)<\varepsilon / 2$. Then there is $\delta^{\prime}>0$ such that if $w, w^{\prime} \in X \times I^{n}$, $d\left(w, w^{\prime}\right)<\delta^{\prime}, A \in C\left(X \times I^{n}\right)$, and $w \in A$, then there is $B \in C\left(X \times I^{n}\right)$ such that $w^{\prime} \in B$ and $d_{H}(A, B)<\delta$. Choose $\delta^{\prime \prime}>0$ such that if $z, z^{\prime} \in X \times Q$ and $d\left(z, z^{\prime}\right)<\delta^{\prime \prime}$, then $d\left(p_{n}(z), p_{n}\left(z^{\prime}\right)\right)<\delta^{\prime}$. Let $z, z^{\prime} \in X \times Q, d\left(z, z^{\prime}\right)<\delta^{\prime \prime}$, 
and $z \in A \in C(X \times Q)$. Then there is $B^{\prime} \in C\left(X \times I^{n}\right)$ such that $p_{n}\left(z^{\prime}\right) \in B^{\prime}$ and $d_{H}\left(p_{n}(A), B^{\prime}\right)<\delta^{\prime}$. Let $B=p_{n}^{-1}\left(B^{\prime}\right)$. Then $z^{\prime} \in B$ and $B \in C(X \times Q)$. We also see that

$$
\begin{aligned}
d_{H}(A, B) & =d_{H}\left(A, p_{n}^{-1}\left(B^{\prime}\right)\right) \\
& \leq d_{H}\left(A, p_{n}^{-1}\left(p_{n}(A)\right)\right)+d_{H}\left(p_{n}^{-1}\left(p_{n}(A)\right), p_{n}^{-1}\left(B^{\prime}\right)\right) \\
& <\varepsilon / 2+\varepsilon / 2=\varepsilon .
\end{aligned}
$$

Hence $X \times Q$ has property [K]. As in the proof of $(2.1), \Lambda(X)$ can be embedded in the Hilbert cube $Q$. Consider the embedding $i: \Lambda(X) \rightarrow X \times Q$ defined by $i(\alpha)=(\alpha(0), \alpha)$. Then the map $G$ satisfies $G \cdot i=$ id (see the proof of $(2.1)$ ). By [14, (2.9)], $\Lambda(X)$ has property [K]. Then $T: \Lambda(X) \times[0, \omega(X)] \rightarrow C(X)$, which is defined by $T(\alpha, t)=\alpha \cap \omega^{-1}(t)$, is monotone. By [14, (4.3)], $C(X)$ has property $[\mathrm{K}]$. Similarly, we obtain that $\omega^{-1}(t)$ has property $[\mathrm{K}]$ for any Whitney map $\omega$ for $C(X)$.

(3.4) Problem. If $X$ has property [K], is it true that $X \times I$ has property [K]?

It is known that there is a continuum $X$ such that $X$ has property $[\mathrm{K}]$, but $X \times X$ does not have property $[\mathrm{K}]$ (see $[14,(4.7)])$.

\section{REFERENCES}

1. W. J. Charatonik, Hyperspaces and the property of Kelley, Bull. Acad. Polon. Sci. Sér. Sci. Tech. 30 (1982), 457-459.

2. D. W. Curtis, Application of a selection theorem to hyperspace contractibility, Canad. J. Math. 37 (1985), 747-759.

3. L. Fearnley, Characterizations of the continuous images of the pseudo-arc, Trans. Amer. Math. Soc. 111 (1964), 380-399.

4. S. Ferry, Strongly regular mappings with compact ANR fibers are Hurewicz fiberings, Pacific J. Math. 75 (1978), 373-382.

5. H. Kato, On the property of Kelley in the hyperspace and Whitney continua, Topology Appl. 30 (1988), 165-174.

6. J. L. Kelley, Hyperspaces of a continuum, Trans. Amer. Math. Soc. 52 (1942), 22-36.

7. J. Krasinkiewicz, On the hyperspaces of snake-like and circle-like continua, Fund. Math. 83 (1974), 155-164.

8. P. Krupski, The property of Kelley in circularly chainable and in chainable continua, Bull. Acad. Polon. Sci. Sér. Sci. Tech. 29 (1981), 377-381.

9. W. Kuperberg, Uniformly pathwise connected continua, Studies in Topology, Academic Press, New York, 1975, pp. 315-324.

10. A. Lelek, On weakly chainable continua, Fund. Math. 51 (1963), 271-283.

11. W. Lewis, Continuum theory problems, Topology Proc. 8 (1983), 361-394.

12. S. B. Nadler, Jr., Some basic connectivity properties of Whitney map inverses in $C(X)$, Studies in Topology, Academic Press, New York, 1975, pp. 393-410.

13. _ Hyperspaces of sets, Dekker, New York and Basel, 1978.

14. R. W. Wardle, On a property of J. L. Kelley, Houston J. Math. 3 (1977), 291-299.

15. H. Whitney, Regular families of curves. I, Proc. Nat. Acad. Sci. U.S.A. 18 (1932), 275-278.

Faculty of Integrated ARts and Sciences, Hiroshima University, Higashisenda-Machi, NAKA-KU, Hiroshima 730, JAPAN 